\title{
SENSITIVITY OF PAS HISTOPATHOLOGY STAIN FOR THE DIAGNOSIS OF ONYCHOMYCOSIS AT MULTICENTER TEACHING HOSPITALS, PAKISTAN.
}

\footnotetext{
1. MBBS, BSc, M.Phil. Microbiology Associate Professor

Department of Pathology Quaid-e-Azam Medical College, Bahawalpur, Pakistan.

2. Assistant Professor Department of Histopathology King Edward Medical University Lahore.

3. Assistant Professor

Department of Biochemistry Quaid-e-Azam Medical College, Bahawalpur.
}

Correspondence Address: Dr. Muhammad Wajid Khurshid Sipra Assistant Professor

Department of Pathology Quaid-e-Azam Medical College,

Bahawalpur, Pakistan.

wajidkhurshid@gmail.com

Article received on: 23/10/2018

Accepted for publication: 25/02/2019

Received after proof reading: $31 / 07 / 2019$

\section{Muhammad Wajid Khurshid Sipra1, Zahid Mehmood Akhtar ${ }^{2}$, Tayyaba Batool ${ }^{3}$}

ABSTRACT... The aim of this study was to determine the sensitivity of Periodic acid -Schiff (PAS) staining an early and quick effective diagnostic test of nail clipping with routine tests in the diagnosis of Onychomycosis. The routine gold standard for diagnosis of fungal nail infections has been direct microscopy (KOH mount) and mycological culture which often yield delayed or weak/false negative results. However recent studies have suggested that nail plate biopsy using PAS stain is rapid method of detection that grasped the diagnosis and manage the disease. Study Design: Cross sectional descriptive study. Setting: 320 clinical diagnosed cases of onychomycosis performed at Department of Microbiology, Quaid-e-Azam Medical College Bahawalpur and Department of Histopathology King Edward medical University Lahore. Period: January 2012 to August 2018. Materials and Methods: The parameter looked in the sent investigation were histopathology PAS stain, mycological culture and microscopy. Presences of intensely stained reddish dots or threads like structures in between the cells of nail plate were considered to be positive results on histopathology with periodic acid Schiff (PAS). The microscopic study showed hyphae or spores and growth of organism concluded by morphological colony characteristics on Sabouraud's dextrose agar (SDA) periodically after 4 weeks. Result: out of total 320 cases, $81.25 \%$ was positive for histopathology PAS stain. Culture positivity was $60 \%$ and $\mathrm{KOH}$ mount recovered $52.5 \%$ positive. The combination of PAS stain and culture results showed $90.62 \%$ while Culture and $\mathrm{KOH}$ were $62.5 \%$ in nail clipping specimen. Conclusion: Histopathological PAS technique was found to be more effective than other laboratory methods for the diagnosis of Onychomycosis.

Key words: $\quad$ Fungal Culture, KOH Mount, PAS (periodic acid Schiff) Staining, Sabouraud's Dextrose Agar

Article Citation: Sipra MWK, Akhtar ZM, Batool T. Sensitivity of PAS histopathology stain for the diagnosis of onychomycosis at multicenter teaching hospitals, Pakistan. Professional Med J 2019; 26(8):1213-1217.

DOI: 10.29309/TPMJ/2019.26.08.257

\section{INTRODUCTION}

Onychomycosis is a growing global health problem and dermatophyte, non- dermatophyte, yeast or molds are the causative agents. Infection due to dermatophyte is known as Tinea unguium. The prevalence of the disease is rising over the world and ranges from $2.1 \%$ to $9.1 \% .^{1,2}$

The diagnosis of onychomycosis with routine histopathological H\&E examination are not considered for nail clippings. The sensitivity of PAS (Periodic acid-Schiff) is reported in literature as better then mycological culture and $\mathrm{KOH}$ microscopy. This increasing reliance makes it apparently "gold standard" in diagnosis of onychomycosis. $^{3}$
Clinically, Onychomycosis starts as a yellowish discoloration of the nail that are further driven towards thick, rough, crumb and separated, which ultimately developed into debris that is accumulated under the nail bed. This thickening and dystrophy result in pressure erosions of the nail bed and Hyponychim. ${ }^{4}$

Onychomycosis traditionally refers to the infection caused by non-dermatophytes but increasingly it is being used to denote all fungal infections of the nails. The term Onychomycosis is derived from the Greek word "onyx" a nail and "mykes" a fungus. ${ }^{5}$ which can be classified into various types including i) distolateral subungal onychomycosis 
(DLSO); ii) superficial white onychomycosis (SWO); iii) proximal subungal onychomycosis (PSO); iv) candida onychomycosis (CO); and v) total dystrophic onychomycosis (TDO). ${ }^{6}$ The general risk factors for onychomycosis are nail trauma, diabetes, immunodeficiency, hyperhydrosis, peripheral vascular diseases, male gender, poor hygiene, increasing age and chronic exposure of nails to water in candidal onychomycosis. ${ }^{7}$ Diabetes mellitus increases the risk of onychomycosis twice and HIV patients are found to be $15-40 \%$ as compared with the general population. ${ }^{8}$ There is higher prevalence of dermatophyte in temperate zone and moulds such as aspergillus species and fusarium species found in tropical and subtropical countries. ${ }^{9}$

In childhood, however onychomycosis is rare. It is particularly unusual under 6 years of age. ${ }^{10}$ The incidence reaches $0.5-2.6 \%$ of all children and toenail are affected more commonly than fingernail. ${ }^{11}$

\section{MATERIALS AND METHODS}

Three hundred twenty patients with clinical suspecting the diagnosis of Onychomycosis reporting to Dermatology outpatient department of Bahawal Victoria Hospital Bahawalpur and JPMC Karachi, a tertiary care hospital, were included in this study. Non-probability convenience sampling technique was applied. The study was carried out from January 2012 to August 2018. Clinical evaluation included detailed history of trauma, occupation, sharing of common facilities, personal habits such as smoking and drinking, personal hygiene, hyperhydrosis and different predisposing diseases. The most severely affected nail was selected for specimen collection. ${ }^{12}$ All the three tests (Histopathology PAS stain, $\mathrm{KOH}$ mount microscopy and mycology culture) were included in the study. This study included patients of age group 01 days to 70 years irrespective of genders, suffering more than one nails. Patients with a history of use of antifungal drugs, psoriasis, lichen planus, contact dermatitis and other systemic diseases were excluded from the study. ${ }^{13}$

The specimens comprised of nail clipping immersed in $20 \% \mathrm{KOH}$ were slightly warmed for softening for both low and high power direct microscopy. The presence of fungal elements i.e. hyphae, spores, budding cells and pseudohyphae were noted. ${ }^{14}$ If fungal elements were detected than nail inserted on SDA (Sabouraud's dextrose) agar and incubated at $37^{\circ} \mathrm{C}$. The growth observed periodically for 4 weeks and then follow colony characteristics by cotton blue solution for identification of species. Nail clippings were fixation in $10 \%$ formalin and then it was treated with $4 \%$ phenol for softening. The further processing included dehydration, embedding in paraffin blocks sectioning by microtome machine mounting on slide. PAS staining were performed it showed the presences of intensely stained reddish dots or threadlike structures between the cell of nail plate and were considered to be the positive results. ${ }^{15}$

\section{RESULT}

Out of three hundred twenty patients, 200 cases were male while 120 was female (Figure-1). Their age ranged from 01 days to 70 years. This study observed that 21 to 30 -year age group having more tendency of nail fungal infections that is $50 \%$ (Figure-2). PAS staining histopathological examination revealed positive results in $81.25 \%$ $\%$, while mycological culture showed $60 \%$ and direct microscopy showed $52.5 \%$ results. It was also observed that the combination of PAS and mycological culture revealed $90.62 \%$ positive results respectively while $\mathrm{KOH}$ and mycological culture showed $62.5 \%$ results. (Table-I). The positive culture showed Dermatophyte were $108(56.25 \%)$ while non-dermatophyte was 84 (43.75\%) (Table-II).

PAS staining showing the more accurate for the diagnosis of onychomycosis either single or combination with routine laboratory methods.

\begin{tabular}{|l|c|c|}
\hline \multicolumn{1}{|c|}{ Test } & Positive n (\%) & Negative n (\%) \\
\hline PAS & $260(81.25 \%)$ & $60(18.75 \%)$ \\
\hline Culture & $192(60 \%)$ & $128(40 \%)$ \\
\hline KOH & $168(52.5 \%)$ & $152(47.5 \%)$ \\
\hline PAS and Culture & $290(90.62 \%)$ & $30(9.38 \%)$ \\
\hline KOH and Culture & $200(62.5 \%)$ & $120(37.5 \%)$ \\
\hline
\end{tabular}

Table-I. Description of diagnostic laboratory methods for the diagnosis of onychomycosis $(n=320)$ 


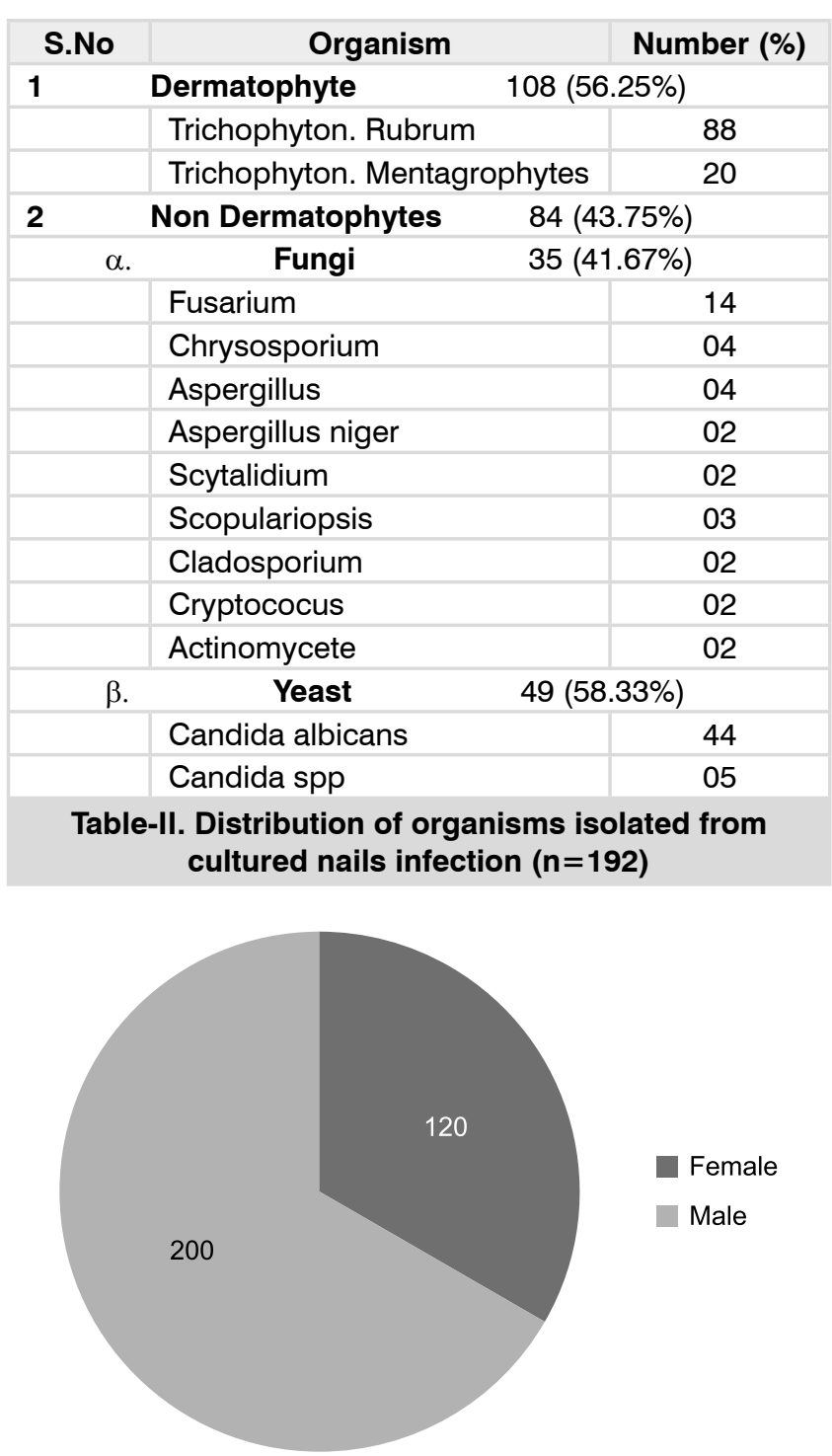

Figure-1. Gender distribution of total study population

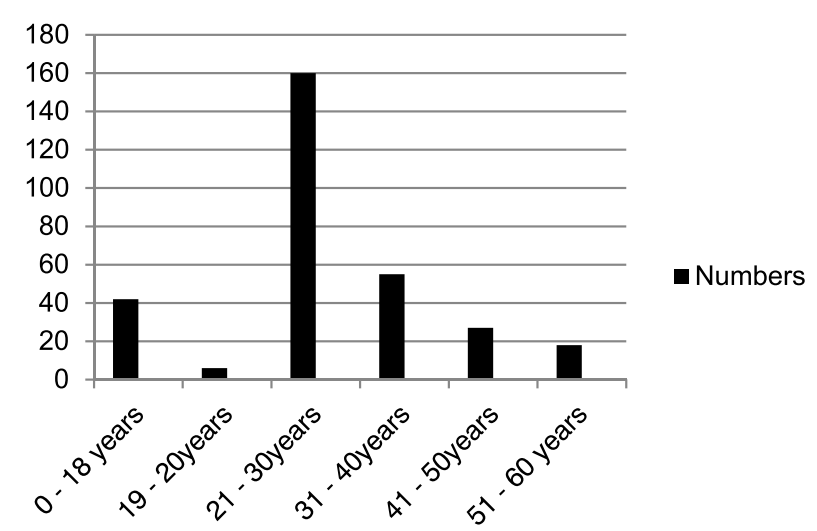

Figure-2. Age distribution of total study population

\section{DISCUSSION}

Onychomycosis is the fungal infection of the nail units because fungi cause partial or whole nail destruction. Theappropriate diagnostic instrument is vital to ensure clinical identification. We aimed to compare histopathology examination-PAS with culture and direct microscopy, for diagnosis of onychomycosis by evaluating their sensitivity. ${ }^{16}$

In this study our results PAS histopathological staining reveal $81.25 \%$ results which is consistent with Shenoy et al. ${ }^{15}$ who showed PAS $75 \%$. Our results are at par with another authors Alkhayat et al. ${ }^{17}$ that showed PAS stain was positive in $79 \%$ cases. Weinberg et al. ${ }^{18}$ that evaluated onychomycosis and found PAS 92\% positive, consistent with our results that is $81.25 \%$. PAS staining by Ahmad $R$ et al. ${ }^{19}$ that showed $77 \%$ results, is consistent with our results. Comparing the direct microscopy, fungal culture and histological staining by Wilsmann-Theis $D$ et al. ${ }^{20}$ concluded that the sensitivity of PAS is $82 \%$ and culture $53 \%$ that are consistent with our results PAS is $81.25 \%$ and culture is $60 \%$ positive. The causative agent for onychomycosis is dermatophyte recovered in our study is $56.25 \%$ matched with $50 \%$ results by Gianni $C$ et al. ${ }^{21}$ Comparing the different Laboratory techniques in our results, PAS showed $81.25 \%$ positive and culture $60 \%$ is consistent with Lilly KK.22 recovered PAS positive is $98.8 \%$ and culture is $57.3 \%$. The study done by Jung MY et al. ${ }^{23}$ that concluded PAS with combination of culture showed $94.1 \%$, and $\mathrm{KOH}$ combination with culture showed $70 \%$ that is consistent with our results that was $90.62 \%$ $\%$ and $62.5 \%$ respectively. The study of Lawry MA et al. ${ }^{24}$ who showed PAS was positive in $85 \%$ and PAS with combination of culture results is $94 \%$ positive that is consistent with our results that was $81.25 \%$ and $90.62 \%$ respectively.

\section{CONCLUSION}

PAS (Periodic acid -Schiff) staining is more efficient techniques for the diagnosis of Onychomycosis than other methods routinely adapted in pathology laboratory.

\section{RECOMMENDATION}

PAS histopathological staining routinely 
performed as a diagnostic tool in pathology laboratory for improving the accuracy for the diagnosis of onychomycosis.

\section{CONFLICT OF INTEREST}

All the cost of the study was born by the $\mathrm{Dr}$ Muhammad Wajid Khurshid Sipra. The authors had no affiliations with any pharmaceuticals/ private organizations during the course of this project.

Copyright $(25$ Feb, 2019.

\section{REFERRENCES}

1. Ahmed R, Kharal S.A, Durrani M.A. Comparison of $\mathrm{KOH}$ mount \& fungal culture in the diagnosis of onychomycosis. PJMH S 2012 Jan-Mar; VOL 6 No 1; 143-8.

2. Bonifaz A, Rios-Yuil J.M, Arenas R, et al; Comparison of direct microscopy, culture and calcofluor white for the diagnosis of Onychomycosis. Rev Iberoam Micol. 2013; 30(2):109-11.

3. D'Hue Z, Perkins S.M, Billings S. D. GMS is superior to PAS for diagnosis of Onychomycosis. J Cutan Pathol. 2008 August; 35(8); 745-47.

4. Rich $P$. Onychomycosis and tinea pedis in patient with diabetes. J Am Acad Dermatol 2000; 3:130-134.

5. Kaur R, Kashyap B, Bhalla P. Onychomycosisepidemiology, diagnosis and management. Indian $\mathrm{J}$ Med Microbiol. 2008 Jun; 26(2):108-16.

6. Lone R, Showkat H. I, Bashir D et al; Clinico-Mycological pattern of onychomycosis A single center one-year study in Kashmir-North India. Eur J Gen Med 2013; 10(3):150-3.

7. Ahmed R, Kharal SA, Durrani MA et al; Frequency of candida in onychomycosis. J Pak Med Assoc. Vol. 63, No.3, March 2013, 350-3.

8. Westerberg D.P, Voyack M.J. Onychomycosis: Current Trends in Diagnosis and Treatment. Indian Journal of Clinical Practice, Vol. 25, No. 4, September 2014.

9. Ellabib M.S, Agaj M, Khalifa Z, and Kavanagh K. Yeasts of the genus Candida are the dominant cause of onychomycosis in Libyan women but not men: Results of a 2-year surveillance study. British Journal of Dermatology 2002; 146: 1038-41.

10. Zisova L, Chokoeva A, et al; Onychomycosis and children a multicenter study. Acta Medica Bulgarica, Vol. XLII, 2015, № 1.
11. Piraccini B.M and Alessandrini A. Onychomycosis: $\mathbf{A}$ Review. J. Fungi 2015, 1, 30-43.

12. Satpathi $P, A c h a r A$, et al; Onychomycosis in Eastern India - study in a peripheral Tertiary Care Centre. Journal of Pakistan Association of Dermatologists 2013; 23 (1):14-19.

13. Agrawal A, Shanker U et al; Clinical and Microbiological study of Tinea unguium in a Tertiary Care Centre. Int. J. Curr. Microbiol. App. Sci (2015) 4(4): 899-905.

14. Ahmad M, Gupta S, Gupte S. A clinico-mycological study of onychomycosis. Egypt Dermatol Online J. 2010 Jun $1 ; 6(4)$.

15. Shenoy M.M, Teerthanath. S, Karnaker V K, et al; Comparison of potassium hydroxide mount and mycological culture with histopathologic examination using periodic acid -Schiff staining of nail clipping in the diagnosis of onychomycosis. Indian $\mathrm{J}$ Dermatol Venereol Leprol May-June 2008, Vol 74 Issue 3, 226-29.

16. Jeelani S, Ahmed QM, Lanker AM, et al. Histopathological examination of nail clippings using PAS staining (HPE-PAS): Gold standard in diagnosis of Onychomycosis. Mycoses, $2015 \mathrm{Jan} ; 58$ (1):27-32.

17. Alkhayat $\mathrm{H}$, Al-Sulaili N, O'Brien, E et al; The PAS stain for routine diagnosis of onychomycosis. Bahrain Medical Bulletin, June 2009; 31 (2), 1-7.

18. Weinberg J M, Koestenblat E K, William D Tutrone W $D$ et al; Comparison of diagnostic methods in the evaluation of onychomycosis. J AM Acod Dermtol 2003:49:193-7.

19. Ahmad R, Kharal SA, Durani MA et al; Comparison of different laboratory methods in the diagnosis of onychomycosis. Ann Abbasi Shaheed Hosp. Karachi, 2010:15(2):127-32.

20. Wilsmann-Theis D, Sareika F, Bieber T, et al; New reasons for histopathological nail-clipping examination in the diagnosis of onychomycosis. J Eur Acad Dermatol Venereol. 2011; 25(2):235.

21. Gianni C, Morelli V, Cerri A, et al; Usefulness of histological examination for the diagnosis of onychomycosis. Dermatology 2001; 202(4):283-8.

22. Lilly KK, Koshnick RL, Grill JP, et al; Cost-effectiveness of diagnostic tests for toenail onychomycosis: a repeated-measure, single-blinded, cross-sectional evaluation of 7 diagnostic tests. J Am Acad Dermatol. 2006; 55(4): 620 . 
23. Jung MY, Shim JH, Lee JH, et al; Comparison of diagnostic methods for onychomycosis, and proposal of a diagnostic algorithm. Clin Exp Dermatol. 2015 Jul; 40(5):479-84.
24. Lawry MA, Haneke E, Strobeck K, et al; Methods for diagnosing onychomycosis: A comparative study and review of the literature. Arch Dermatol. 2000; 136(9):1112.

\section{AUTHORSHIP AND CONTRIBUTION DECLARATION}

\begin{tabular}{|c|c|c|c|}
\hline Sr. \# & Author-s Full Name & Contribution to the paper & Author $=$ s Signature \\
\hline 1 & M. Wajid Khurshid Sipra & $\begin{array}{l}\text { Conceive of idea, Writing of } \\
\text { manuscripts, Interpretation } \\
\text { of results, Final review of } \\
\text { manuscripts. }\end{array}$ & \\
\hline 2 & Zahid Mehmood Akhtar & $\begin{array}{l}\text { Interpretation of Resutls, Final } \\
\text { review of manuscript. }\end{array}$ & \\
\hline 3 & Tayyaba Batool & $\begin{array}{l}\text { Interpretation of Resutls, Final } \\
\text { review of manuscripts. }\end{array}$ & \\
\hline
\end{tabular}

